\title{
Meta
}

Journal des traducteurs

Translators' Journal

\section{CASTRO RAMÍREZ, Nayelli, dir. (2013) : Traducción, identidad y nacionalismo en Latinoamérica. Mexico : Bonilla Artiga, 286 p.}

\section{Raúl Ernesto Colón Rodríguez}

Volume 62, numéro 3, décembre 2017

URI : https://id.erudit.org/iderudit/1043960ar

DOI : https://doi.org/10.7202/1043960ar

Aller au sommaire du numéro

Éditeur(s)

Les Presses de l’Université de Montréal

ISSN

0026-0452 (imprimé)

1492-1421 (numérique)

Découvrir la revue

Citer ce compte rendu

Colón Rodríguez, R. E. (2017). Compte rendu de [CASTRO RAMíREz, Nayelli, dir. (2013) : Traducción, identidad y nacionalismo en Latinoamérica. Mexico :

Bonilla Artiga, 286 p.] Meta, 62(3), 657-660. https://doi.org/10.7202/1043960ar

Ce document est protégé par la loi sur le droit d'auteur. L'utilisation des services d’Érudit (y compris la reproduction) est assujettie à sa politique d'utilisation que vous pouvez consulter en ligne.

https://apropos.erudit.org/fr/usagers/politique-dutilisation/ 
de sus vínculos con la intersubjetividad, el autor reflexiona sobre el espacio público-político, la comunicación oral-escrita y la frontera en las relaciones hispano-mapuches, con fin de repensar la convivencia en el mundo actual. El artículo de Francisco Raga Gimeno «En el corazón de la cultura. Hibridación y mediación intercultural en el ámbito de la salud mental», aborda el concepto de interculturalidad y sus vínculos con la interpretación en el campo psiquiátrico. Subraya el carácter cultural de este mismo y sugiere implantar lo que llama «espacios interculturales más integrales». El artículo de María López Ponz «From la otra orilla and back: representaciones del mestizaje en las traducciones al español de literatura hispanoestadounidense» trata de las estrategias utilizadas, en las traducciones españolas de obras de escritoras hispano-estadounidenses, para representar las diferentes formas de spanglish y los elementos biculturales y de cómo la posición y el habitus de los traductores influye en este proceso. Juan Jesús Zaro propone en su trabajo "La traducción de Shakespeare en la América de lengua española: entre la tradición y la transculturación», explorar las diferentes traducciones en español del autor a partir de una clasificación que distingue las traducciones tradicionales, de las transculturadas. Finalmente, el epílogo de la presente obra está constituido por la contribución de Yolanda Onghena «De contexto a contexto: movilidades transnacionales» que aborda la tensión identidaddiferencia en los contextos migratorios y propone aceptar la naturaleza dinámica del proceso identificación-diferenciación.

En resumen, Traducción y representaciones del conflicto desde España y América. Una perspectiva interdisciplinar propone un recorrido histórico polifacético de algunas prácticas e instituciones de mediación intercultural e interlingüísticas, que existieron a partir del siglo IV hasta la actualidad. La fuerza de esta recopilación radica, en nuestra opinión, en su planteamiento interdisciplinar que permite aprehender este mismo objeto a partir de las perspectivas de campos tan variados como la traductología, la historia, la antropología, la filosofía y la literatura. Esta aproximación innovadora presenta un panorama amplio y nos permite apreciar las distintas manifestaciones del fenómeno conflicto-mediación en toda su complejidad.

Sin que la calidad de las reflexiones expuestas se viera afectada, no podemos obviar que son las miradas españolas las que siguen dominando y que las miradas americanas se originan exclusivamente desde Chile y Argentina, y eso a pesar de la crítica del etnocentrismo que atraviesa todo el libro. Si, por una parte, el lugar en que se llevó al cabo el seminario del cual deriva este libro y el lugar de procedencias de los tres grupos involucrados ofrece una explicación satisfactoria, confiamos en que la inclusión de otras voces marginadas hubiera encajado perfectamente con el planteamiento de este proyecto y enriquecido todavía más el horizonte presentado.

Finalmente, el eclecticismo de los artículos, que resulta no precisamente del enfoque multidisciplinario, sino de la multiplicidad de los objetos, subtemas y perspectivas, así como del amplio periodo cubierto, constituye a la vez parte de la originalidad de la presente obra y su límite. Sin embargo, el hecho que se puede adquirir el ebook por capítulos no dejará de atraer académicos interesados solamente en uno $\mathrm{u}$ otro de los subtemas desarrollados.

\section{Anne-Marie Gagné Université de Montréal, Montreal, Canadá}

\section{REFERENCIAS}

BAker, Mona (2006): Translation and Conflict. A Narrative Account. Londres/Nueva York: Routledge.

Steiner, George (1975): After Babel. Oxford: Oxford University Press.

CAstro Ramírez, Nayelli, dir. (2013): Traducción, identidad y nacionalismo en Latinoamérica. Mexico: Bonilla Artiga, 286 p.

Le tournant de 2014 a été faste pour les publications sur l'histoire de la traduction en Amérique hispanique. En octobre 2013, le premier Diccionario histórico de la traducción en Hispanoamérica voit le jour en Espagne, comme suite logique et attendue du Diccionario histórico de la traducción en España (2009), tous deux coordonnés par Francisco Lafarga et Luis Pegenaute ${ }^{1}$. Au Mexique, en juin 2014², paraît Traducción, identidad y nacionalismo en Latinoamérica, ouvrage collectif dirigé par Nayelli Castro Ramírez, que nous abordons ici. Malgré le titre inclusif, qui autoriserait la présence de contributions sur le Brésil, l'ouvrage ne compte que des chapitres sur des sujets hispano-américains. Néanmoins, cette publication constitue, à n'en pas douter, un premier et louable effort d'historiciser la traduction de manière continentale, selon plusieurs axes. Ce texte est d'ores et déjà inclus dans la bibliographie de cours de traduction en Amérique hispanique . $^{3}$

La comparaison entre les perspectives latinoaméricaines $^{4}$ de facture mexicaine et le Dictionnaire historique de facture espagnole est inévitable, parce que les deux ouvrages contribuent à ouvrir en mode collectif, dans le traditionnel format du livre imprimé, le champ de la recherche traductologique, qui est celui de l'histoire de la traduction en 
Amérique hispanique ou, si l'on veut, en Amérique latine. Le développement de ce champ a été jusqu'ici plutôt le résultat des efforts de chercheurs individuels, exception faite du groupe de recherche sur l'Histoire de la traduction en Amérique latine (HISTAL) de l'Université de Montréal qui, depuis 2004, compile et tient à jour une encyclopédie en ligne sur l'histoire de la traduction en Amérique latine ${ }^{5}$.

La différence fondamentale entre l'ouvrage espagnol et le mexicain réside dans l'envergure des deux projets. Au premier ont collaboré plus d'une centaine de chercheurs du monde entier (dont plusieurs qui ont ensuite participé à l'ouvrage mexicain). Il compte 214 entrées et bénéficie d'outils d'indexation qui permettent une consultation rapide dans dix-neuf groupes d'entrées par pays (ámbitos ou milieux géopolitiques), y compris Puerto Rico.

L'ouvrage mexicain repose sur 10 collaborateurs, en plus de la coordonnatrice Castro Ramírez. Il a été conçu suivant une approche thématique et chronologique plus traditionnelle, et concentre son attention sur quelques pays hispano-américains, notamment le Mexique, la Colombie, le Chili et le Venezuela. Le texte est organisé en trois sections ou chapitres intitulés «Avatars traductifs de la colonie aux indépendances», "Forger la nation: traduire pour éduquer» et «Conjonctures actuelles: traduction et interculturalité ${ }^{6}$. L'actualisation théorique et la description de l'état actuel des études en histoire de la traduction en Amérique latine ou sur cette région sont traitées dans plusieurs des articles de cet ouvrage collectif. Ceci est particulièrement probant dans la présentation de l'ouvrage, où la coordonnatrice nous offre, en plus d'une description du contenu du livre, un compte rendu des développements théoriques et épistémologiques récents en histoire de la traduction, ainsi qu'un rappel des récents congrès et colloques sur le sujet. Ces événements ont culminé par la fondation du Réseau latino-américain des études de la traduction et de l'interprétation (RELAETI) ${ }^{7}$ qui devrait relancer l'intérêt et la reconnaissance de la traductologie dans la région.

La première section de Traducción, identidad y nacionalismo en Latinoamérica touche plusieurs sujets liés à la traduction lors de la transition entre l'époque coloniale et les indépendances hispanoaméricaines. Les articles de cette section ciblent un cadre national, par exemple celui de Payàs sur le Chili, qui comporte aussi des réflexions théoriques sur l'utilité révisionniste de l'histoire de la traduction versus l'Histoire en général. Bastin, Echeverri et Campo étudient pour leur part l'importance historique et certainement idéologico-politique de la traduction par le colombien Antonio Nariño de La Déclaration des droits de l'homme et du citoyen de 1789, et de la Lettre aux Espagnols Américains de Juan Pablo Viscardo y Guzmán publiée en 1792 par le Vénézuélien Francisco de Miranda, tous les deux des documents clés dans les processus indépendantistes hispano-américains. Navarro se concentre sur un support spécifique - la presse pour montrer que l'appropriation de l'original en Amérique hispanique s'est faite sur les deux versants du spectre politique. Ces deux derniers articles s'étendent sur un cadre hispano-américain plus large, en plus de faire des incursions extracontinentales. Ces trois articles relèvent de chercheurs qui travaillent ou ont été formés dans l'espace traductologique canadien, ce qui fait état de l'influence de ce pays dans le développement de la discipline en Amérique latine.

La deuxième section de l'ouvrage collectif est consacrée au rôle de la traduction dans les processus de mise en place du credo nationaliste par le biais de l'éducation, et ce, tant en Colombie qu'au Mexique. L'article de Montoya Arango consiste en une excellente introduction sur l'histoire de la traduction en Colombie, et par ricochet en Amérique latine. Les articles de González Kahn et de Castro Ramírez versent pour leur part sur le Mexique. Ces auteures nous parlent des traductions promues par José Vasconcelos, homme politique mexicain du début du $\mathrm{xx}^{\mathrm{e}}$ siècle et ministre de l'Éducation de 1921 à 1924. On y explore la traduction de textes philosophiques de l'Antiquité au Mexique après la révolution de 1910. Ces trois contributions mettent l'emphase sur les particularités des institutions nationales, des personnalités politiques et des réseaux intellectuels qui œuvrent, à travers la traduction, à la consolidation ces deux États-nations en formation. Une analyse critique du paradigme nationaliste demeure absente de ces études, paradigme qui est encore perçu comme «naturel» en traductologie. Toutefois, des traductologues se sont déjà attaqués à des paradigmes de la traduction longtemps considérés inamovibles, par exemple celui considérant le littéralisme et la traduction libre comme les seules approches possibles, donc «naturelles» jusqu'aux années 1960 (Genztler 1993; Munday 2001). Il serait peut-être temps, en histoire de la traduction, de commencer à critiquer ce «naturel» associé au nationalisme, d'autant plus que la condition du traducteur, et souvent celle du traductologue, a été et reste pionnière dans la contestation de cette idéologie moderne. Le «moi» identitaire du traducteur ou du traductologue n'est pas monolithique, mais plutôt dynamique, comme le montrent les biographies de la plupart des auteurs du collectif ici recensé. Sur la critique du nationalisme, soulignons l'ouvrage sur l'anthropologie du nationalisme de Claudio Lomnitz (2001), aujourd'hui un classique sur cette question, l'espace public et la construction des connaissances au Mexique. Notons également la 
critique du paradigme nationaliste dans un travail collectif sur les mobilités culturelles, en particulier la contribution de Pierre Ouellet (2014:367), pour qui la mobilité n'est "plus seulement transnationale ou transculturelle», mais "transsubjective», de même que celle d'Alexis Nuselovici (2014).

L'axe central de la troisième section est «l'hétérogénéité culturelle propre aux sociétés contemporaines en Amérique latine» (p. 17), analysée à partir de la traduction de documents propres au contexte mexicain. Dans le premier article, D’Amore prône la glose en tant que recours d'approximation à l'étrangeté. Ceci permettrait d'expliciter les nuances culturelles et raciales dans la traduction anglaise des surnoms couramment utilisés dans la vie sociale et politique mexicaine ou latino-américaine, surnoms qui se trouvent souvent dans la littérature des auteurs de ce pays. Zaslavsky, doyenne des études de la traduction au Mexique, traite un sujet d'actualité politique au Mexique, soit le conflit traduction-interprétation en lien avec un cas policier en milieu aborigène. Cette étude présente par ailleurs un problème théorique intéressant que l'auteure résume brièvement comme «la représentation que l'on veut apporter de la traduction comme activité et comme produit», c'est-à-dire "les conditions réelles d'inégalité dans lesquelles ont lieu les traductions-interprétations en contexte juridique " (p. 243-244). Limón Aguirre touche aussi le sujet de la traduction et des populations aborigènes du Mexique. Cet auteur prône une traduction constructrice d'interculturalité dans ces contextes, ce qui témoigne de l'intérêt croissant au Mexique pour la thématique aborigène ${ }^{8}$. Notons toutefois une utilisation réduite des sources théoriques disponibles en traductologie dans cette section. L'absence de réflexion sur la théorie du polysystème d'Even-Zohar est à regretter puisque celui-ci a traité en profondeur la question de l'hétérogénéité culturelle et son importance dans des sociétés bilingues ou multilingues (Even-Zohar 2005: 3-4). En effet, la plupart des sociétés latino-américaines, tout particulièrement le Mexique, sont dans cette situation en raison du poids linguistique et culturel de leur composante aborigène.

Bien que l'ouvrage comporte une table des matières et deux pages de biobibliographies, il aurait eu avantage à offrir des index plus complets, par exemple la liste des auteurs et des traducteurs mentionnés dans les articles, de même qu'un index des sujets traités. Ces outils sont aujourd'hui courants, voire indispensables, en histoire de la traduction et dans les publications traductologiques en général.

Traducción, identidad y nacionalismo en Latinoamérica est un ouvrage de consultation recommandé à tous ceux qui s'intéressent à l'his- toire de la traduction en Amérique latine, en particulier en Amérique hispanique. Il est aussi un point de départ pour des recherches et des publications qui, souhaitons-le, complémenteront ce louable effort.

Raúl ERnesto Colón Rodríguez Université d'Ottawa, Ottawa, Canada

\section{NOTES}

1. Voir Colón Rodríguez, Raúl (2015): Compte rendu de Lafarga, Francisco et Pegenaute, Luis, dir. (2013): Diccionario histórico de la traducción en Hispanoamérica. Madrid/ Frankfurt am Main: Iberoamericana/ Vervuert, 515 p. Meta. 60(1):201-204.

2. Le site de l'ALAETI a annoncé la sortie de l'ouvrage en février 2014; il a été lancé le 21 juin 2014 à la librairie de la maison d'édition Bonilla.

3. C'est le cas du cours Traducción y mediación lingüística. Historia y perspectivas latinoaméricanas, de l'Universidad de Chile.

4. Le sous-titre de la présentation de l'ouvrage, soit Estudios históricos de la traducción: Perspectivas latinoamericanas, est juste puisque parmi les auteurs se trouvent non seulement des auteurs de l'Amérique hispanique, mais également du Canada.

5. Voir le site du groupe de recherche sur l'Histoire de la traduction en Amérique latine (HISTAL): www.histal.ca.

6. Nos traductions. Titres originaux: «Avatares traductores de la colonia a las independencias», "Forjar la nación: traducir para educar» et "Coyunturas actuales: traducción e interculturalidad». Toutes les traductions vers le français sont les nôtres, sauf indication contraire.

7. Red Latinoamericana de Estudios de la Traducción e Interpretación / Rede LatinoAmericana de Estudos de Tradução e Interpretação. Voir http://relaeti.org.

8. À titre d'exemple, notons le congrès La etnografía y los desafíos del México contemporáneo: Etnografía de las regiones indígenas de México en el nuevo milenio tenu en octobre 2014 dans la capitale mexicaine et qui traitait tout particulièrement de la pluriculturalité et du plurilinguisme du peuple mexicain.

\section{RÉFÉRENCES}

Even-Zohar, Itamar (2005): Polysystem Theory (revised). In: Itamar Even-Zohar. Papers in Culture Research. Tel Aviv: Porter Chair of Semiotics.

Gentzler, Edwin (1993): Contemporary Translation Theories. Londres/New York: Routledge. 
Lafarga, Francisco et Pegenaute, Luis, dir. (2009): Diccionario histórico de la traducción en España. Madrid: Editorial Gredos.

Lafarga, Francisco et Pegenaute, Luis, dir. (2013): Diccionario histórico de la traducción en Hispanoamérica. Madrid/Frankfurt am Main: Iberoamericana/Vervuert.

Lomnitz, Claudio (2001): Deep Mexico, Silent Mexico: An Anthropology of Nationalism. Minneapolis: University of Minnesota Press.

Munday, Jeremy (2001): Introducing Translation Studies: Theories and Applications. Londres/ New York: Routledge.

Nuselovici, Alexis (2014): Non-lieux (une atypologie). In: Zilá Bernd et Norah Dei CAsGIRALDI, dir. Glossaire des mobilités culturelles. Berne: Peter Lang, p. 257-274.

Ouellet, Pierre (2014): Transportation. In: Zilá Bernd et Norah Dei Cas-Giraldi, dir. Glossaire des mobilités culturelles. Berne: Peter Lang, p. 354-367.

Baumgarten, Stefan and Gagnon, Chantal (2016): Translating the European House. Discourse, Ideology and Politics - Selected Papers by Christina Schäffner. Newcastle: Cambridge Scholars, $382 \mathrm{p}$.

Translation Studies (TS) is currently in the midst of a gradual generational change. Some of the most decisive scholars of the $20^{\text {th }}$ century are now retiring. Following in the footsteps of previous generations, these scholars are largely responsible for the independence of our discipline, its good epistemological health, its hybridity, and its creativity - in sum, its current status quo. They are also largely responsible for leaving behind a cohort of well-prepared academics. When masters give way, their disciples would do well to pause and review their roots before (hopefully) taking a leap forward. The present book, Translating the European House, is Stefan Baumgarten and Chantal Gagnon's review of the work of an essential translation studies scholar, their $\mathrm{PhD}$ supervisor Christina Schäffner.

Baumgarten and Gagnon's contribution here is threefold. First, they have prepared two introductory chapters to the volume ("General Introduction" and "Political discourse analysis in a multilingual world"), in which they review Schäffner's life and works and put both of them into context. Second, they have selected twelve of Schäffner's most well-known papers on the topic of political discourse, presented in chronological order (chapters I to XII). Third, they have compiled Schäffner's bibliography on the topic listed from 2016 to 1981 ("Publications by Christina Schäffner").
In the "General Introduction," Baumgarten and Gagnon focus on Schäffner's life. Originally from East Germany (Schlotheim), Schäffner started her academic career in 1969 at the then Karl Marx University (Leipzig). They follow her life to the culmination of her work in Translation Studies to where she has recently been awarded the title of Emeritus Professor at Aston University (Birmingham, UK). That the authors start the volume with some lines on Schäffner's life is a felicitous decision. It is precisely her evolved position from Eastern Europe to the Western World, from the then "ostracised" Germanic tradition to the Anglo-Saxon epistemological hegemony, which gives her work a special resonance. With this position in life, it is no wonder Schäffner devotes her time to political discourse analysis, to multilingual and multicultural dissections and recontextualisations, to what she aptly calls "Critical Translation Studies" (p. 283) and to a united Europe, of course. It is no wonder either that she builds her examinations upon the frameworks developed by her predecessors and contemporaries at Western (often Anglo-Saxon) centres (Fillmore, Lakoff and Johnson, Fairclough) without abandoning her Leipzig text-linguistic training (Neubert). At the same time, she is open to the new developments being put forward by (young) Asian scholars (e.g., Kang). This book is a clear reminder of the fact that, in the same way Schäffner has accepted the challenge to contribute to making Translation Studies larger and stronger from her own vital stance (with her past influences and her newly acquired intertextual and interdiscursive inspirations), it is now time for a new generation to take a step forward and accept responsibility for performing research in a post-Brexit, post-truth world.

In the second introduction to the volume "Political discourse analysis in a multilingual world," Baumgarten and Gagnon perform three exercises of what they label "recontextualised recontextualization" (p. 5) along three dimensions (discursive, communicative and translational), around which Schäffner's work revolves. With these exercises, the editors re-locate her "ideas and favoured concepts" (p. 5). In the first dimension, "International Security and Peace," they point at discursive notions as taken from Fairclough ("discourse," "social practice," "discoursal practice," "text," etc.) and Bourdieu ("political field," "political responsible agents," etc.), which serve as the platform upon which to articulate other typically Schäffnerian concerns, such as "recontextualization," "intertextuality," "interdiscursivity" and "a free-floating web of discursive (i.e., thematic, attitudinal and linguistically inflected) constellations that are habitually invoked by political actors" (p. 5). In the second dimension, "Political 